\title{
BMJ Open Psychosocial treatments for relapse prevention in schizophrenia: study protocol for a systematic review and network meta-analysis of randomised evidence
}

$\overline{\text { Irene Bighelli (D) , }{ }^{1} \text { Alessandro Rodolico, }{ }^{2} \text { Gabi Pitschel-Walz, }{ }^{1} \text { Wulf-Peter Hansen, }{ }^{3}}$ Corrado Barbui, ${ }^{4}$ Toshi A Furukawa, ${ }^{5}$ Georgia Salanti, ${ }^{6}$ Stefan Leucht ${ }^{1}$

To cite: Bighelli I, Rodolico A, Pitschel-Walz G, et al. Psychosocial treatments for relapse prevention in schizophrenia: study protocol for a systematic review and network metaanalysis of randomised evidence. BMJ Open 2020;10:e035073. doi:10.1136/ bmjopen-2019-035073

- Prepublication history for this paper is available online To view these files, please visit the journal online (http://dx.doi. org/10.1136/bmjopen-2019035073).

Received 17 October 2019 Revised 02 December 2019 Accepted 05 December 2019

Check for updates

(C) Author(s) (or their employer(s)) 2020. Re-use permitted under CC BY-NC. No commercial re-use. See rights and permissions. Published by BMJ.

For numbered affiliations see end of article.

Correspondence to

Dr Irene Bighelli;

irene.bighelli@tum.de

\section{ABSTRACT}

Introduction There is evidence that different psychosocial interventions could reduce the risk of relapse in schizophrenia, but a comprehensive evidence based on their relative efficacy is lacking. We will conduct a network metaanalysis (NMA), integrating direct and indirect comparisons from randomised controlled trials (RCTs) to rank psychosocial treatments for relapse prevention in schizophrenia according to their efficacy, acceptability and tolerability.

Methods and analysis We will include all RCTs comparing a psychosocial treatment aimed at preventing relapse in patients with schizophrenia with another psychosocial intervention or with a no treatment condition (waiting list, treatment as usual). We will include studies on adult patients with schizophrenia, excluding specific subpopulations (eg, acutely ill patients). Primary outcome will be the number of patients experiencing a relapse. Secondary outcomes will be acceptability (dropout), change in overall, positive, negative and depressive symptoms, quality of life, adherence, functioning and adverse events. Published and unpublished studies will be sought through database searches, trial registries and websites. Study selection and data extraction will be conducted by at least two independent reviewers. We will conduct random-effects NMA to synthesise all evidence for each outcome and obtain a comprehensive ranking of all treatments. NMA will be conducted in $\mathrm{R}$ within a frequentist framework. The risk of bias in studies will be evaluated using the Cochrane Risk of Bias tool and the credibility of the evidence will be evaluated using Confidence in Network Meta-Analysis (CINeMA). Subgroup and sensitivity analyses will be conducted to assess the robustness of the findings. Ethics and dissemination No ethical issues are foreseen. Results from this study will be published in peer-reviewed journals and presented at relevant conferences. PROSPERO registration number CRD42019147884.

\section{INTRODUCTION}

Schizophrenia is characterised by acute episodes often followed by symptom improvement, ${ }^{1}$ but it requires generally maintenance treatment in order to prevent recrudescence of symptomatology.
Strengths and limitations of this study

- This will be the first network meta-analysis (NMA) on psychosocial treatments for relapse prevention in schizophrenia; the findings from this study have the potential to inform and influence clinical decisionmaking and guideline development.

- The analysis will benefit from maximum statistical power by combining direct and indirect comparisons in a NMA, measuring the relative effects of the different treatments.

- There is risk of heterogeneity and inconsistency, given the different psychosocial interventions that will be included; however, we try to control variability by carefully framing the inclusion criteria about population, interventions and focus of the single studies, and we will evaluate consistency employing local as well as global methods.

- The limitations of individual studies will be addressed with the Cochrane risk of bias tool and the credibility of the results for the primary outcome will be assessed using the Confidence in Network Meta-Analysis web application, an adaptation of the GRADE framework; these approaches are considered the gold standard for critical appraisal of evidence quality.

A 5-year analysis of 11291 patients with schizophrenia found a $13.4 \%$ rehospitalisation rate within 1 month, $38.9 \%$ within 1 year and $64.1 \%$ within 5 years. ${ }^{2}$

Pharmacological interventions have been the mainstay of treatment for schizophrenia, and they also play an essential role in the prevention of relapses. According to a recent meta-analysis of 65 randomised trials, patients treated with antipsychotics experienced a psychotic relapse within 1 year in $27 \%$ of the cases. $^{3}$

Moreover, antipsychotics have a number of limitations (high incidence of disabling side 
effects, poor adherence to treatment $)^{3}$ and can be problematic in many situations (such as medical comorbidities, tolerability problems and pregnancy).

The resulting burden for patients, relatives and society is dramatic because relapses often lead to costly hospitalisations; patients lose their jobs and relationships are challenged. $^{45}$

In this context, psychosocial interventions might have an important role to reduce the risk of relapses.

Several systematic reviews and meta-analyses have examined the comparative efficacy and acceptability of psychosocial interventions from randomised controlled trials (RCTs) in schizophrenia considering relapses among other outcomes. Different interventions such as family therapy, ${ }^{6}$ psychoeducation ${ }^{78}$ and cognitive behavioural therapy ${ }^{9}{ }^{10}$ have been compared with the so-called no treatment conditions (waiting list, treatment as usual (TAU) $),{ }^{79}$ and in some cases also compared with other psychosocial treatments pooled together, ${ }^{6}{ }^{10}$ showing promising results.

A review of 25 studies examining family interventions found a $20 \%$ reduction of relapse when involving the relatives in the treatment during maintenance phase. ${ }^{6}$

Psychoeducation was found to be successful in reduction of relapses in a Cochrane review analysing 11 studies in the medium term and six studies in the long term comparing psychoeducation with standard care (RR $0.70,95 \%$ CI 0.61 to 0.81 ; RR $0.73,95 \%$ CI 0.62 to 0.85$)^{7}$; brief psychoeducation also showed positive results in the medium term for people with severe mental illnesses. ${ }^{8}$ Cognitive behavioural therapy was found to reduce relapse rates in the medium term when compared with standard care, ${ }^{9}$ but not when compared with other psychosocial therapies. ${ }^{10}$

However, evidence is still fragmentary and a comprehensive ranking of all treatments evaluated in RCTs is lacking.

In fact, all the available reviews applied pairwise metaanalysis as a method, and they can therefore provide information only on comparisons of two treatments that have been considered in existing studies, and several interventions lack of head-to-head comparisons. For example, both individual psychoeducation and family interventions have shown efficacy in the reduction of relapses, but the two have never been compared with each other.

As a result, it is still unclear which are the most efficacious, the most acceptable and the best tolerable psychosocial treatments for relapse prevention in schizophrenia. Better understanding of the comparative efficacy of these active treatments would be important for clinical practice and for meaningful allocation of resources.

To overcome this gap in the current knowledge, we will perform a network meta-analysis (NMA) comparing all the interventions with each other and produce hierarchies of the effects of the various psychosocial treatments. Such hierarchies are essential for guidelines, which should ideally be able to indicate which treatment is likely to be the best, the second best and so on for a given outcome.
Only the method of NMA can provide such hierarchies by combining all the randomised evidence.

\section{Objectives}

To estimate relative treatment effects and obtain a hierarchy for the psychosocial treatments for relapse prevention in patients with schizophrenia, in terms of:

1. Relapse and hospitalisations.

2. Other efficacy measures: overall symptoms, positive symptoms, negative symptoms, depressive symptoms, quality of life, adherence, overall functioning.

3. Acceptability (dropout) and tolerability (adverse events).

\section{METHODS AND ANALYSIS}

Methods for this systematic review have been developed according to the Preferred Reporting Items for Systematic review and Meta-Analysis Protocols (PRISMA-P) checklist, and the PRISMA extension statement for reporting of systematic reviews incorporating network meta-analyses of healthcare interventions. ${ }^{11} 12$ This systematic review and NMA is registered in the PROSPERO database (registration number: CRD42019147884). The record in PROSPERO will be updated with any amendment made to the protocol. The methods are consistent with the protocol of a previous NMA by our group on psychological intervention for the positive symptoms of schizophrenia. ${ }^{13}$

\section{Criteria for considering studies for this review \\ Types of studies}

RCTs will be included. We will accept open and blinded RCTs; this choice is particularly relevant in trials on psychological interventions, in which only the assessor of outcome can be blind, but not the clinicians providing the intervention. The effect of a non-blind assessor will be examined excluding these studies in a sensitivity analysis. In the case of cross-over studies, we will use only the first cross-over phase in order to avoid the problem of carry-over effects which are very likely in schizophrenia and with psychosocial treatments. Studies described as randomised, but in which a closer evaluation with the RoB V.2.0 leads to a 'high risk of bias' judgement in the domain 'risk of bias arising from the randomization process', will be excluded. There will be no language restriction in order to avoid the problem of 'language bias'. ${ }^{14}$ Studies will be considered for inclusion irrespective of setting (inpatients or outpatients), participant gender, nationality and ethnicity.

For all the selection criteria (participants, interventions, comparators, outcomes, study design), we will pay attention to the joint randomisability of the interventions for the studied populations namely if the included participants are in principle jointly randomisable to a hypothetical huge trial comparing all the included interventions.

\section{Types of participants}

Individuals aged 18 years or older with a diagnosis of schizophrenia or related disorders (schizophreniform 
or schizoaffective disorders); there is no clear evidence that the latter schizophrenia-like psychoses are caused by fundamentally different disease processes or require different treatment approaches. ${ }^{15}$

We will include trials irrespective of the diagnostic criteria used. Here we will follow the strategy of the Cochrane Schizophrenia Group ${ }^{16}$ to include not only the studies that used specific diagnostic criteria such as ICD-10 or DSM-V, because these criteria are not meticulously used in clinical routine either. This decision should increase generalisability and representativeness.

Studies including participants with other diagnoses part of the psychosis spectrum will be included only if participants with a diagnosis of schizophrenia, schizophreniform or schizoaffective disorders were more than $80 \%$ of the participants considered.

We will exclude studies where all patients, according to the study inclusion criteria, (1) are acutely ill (eg, showing agitation/aggression), but we will include studies if the description of the treatment implies that they are stable enough to receive the intervention (eg, psychoeducation initiated during hospital stay, but it is clear it will be offered to the patients when they are stable enough to take part in the sessions), (2) have comorbid psychiatric disorders, including substance abuse, (3) have a concomitant medical illness, (4) are prodromal or 'at risk for psychosis'.

\section{Types of interventions}

We will include any psychosocial intervention whose main target in the included study is relapse prevention. We expect to include specific psychotherapies (eg, cognitive behavioural therapy designed for relapse prevention, compliance therapy), non-specific psychotherapies (eg, supportive therapy), group psychotherapies (eg, family intervention, psychoeducation), interventions focused on psychosocial functioning (eg, social skills training), interventions including the broader context in which the patient lives (eg, case management and assertive community treatment).

The interventions mentioned above are typical examples. Nevertheless, if during the screening process we identify studies meeting inclusion criteria that examine other interventions we will include them as long as they are deemed jointly randomisable with those mentioned above.

The interventions can be of any length. Psychosocial treatments as defined above will be compared with each other and to any non-pharmacological control condition. Control conditions will include no additional treatment (eg, TAU), waiting list and inactive treatments (eg, psychological placebo). When TAU is used as a waiting list, we will classify this condition as waiting list.

\section{Outcome measures}

\section{Primary outcome}

Our primary outcome is relapse. In case more measures of relapse are reported, we will give priority to measures defined in the following order: (1) operationalised criteria, (2) psychiatric hospital admissions, (3) clinical judgement and (4) need for additional medication or for extra psychotherapy sessions/meetings with the therapist. Other definitions that we cannot foresee at the protocol stage will also be discussed and considered for inclusion. We will extract the number of patients who relapsed and the definition that was used by the authors.

Dropouts will be considered as having relapsed in a sensitivity worst case scenario analysis, unless it is clear that they have already been counted among relapsed patients by the authors of the study.

Primary outcome relapse will be reported separately up to 6 months (26 weeks), up to 12 months (between 26 and 52 weeks-primary time point), more than 12 months.

We want to include studies in which the psychosocial treatment aims at preventing relapse. Therefore, studies will be included if relapse or rehospitalisation is measured among the primary outcomes according to the protocol or methods of the trial.

Where not explicitly reported in the study methods or protocol, the judgement about whether relapse or rehospitalisation can be considered as primary or co-primary outcome will be made observing:

- Whether it is mentioned in the title (eg, 'psychoeducation for relapse prevention in schizophrenia').

- Whether it is the only outcome measured in the study.

- Whether it is declared among study aims (eg, "the main objective of this study was to examine the efficacy of family intervention to prevent rehospitalisation').

- Whether the power calculation was planned to detect differences in the outcome relapse or rehospitalisation.

Studies in which there is a declared primary outcome other than relapse or rehospitalisation will be excluded. The exact criterion used for the judgement about inclusion of each study will be reported for the seek of transparency.

For a study to be included, the assessment of the outcome must have been performed at minimum 12 weeks from randomisation.

\section{Secondary outcomes}

1. Acceptability: number of premature discontinuation ('dropout'), reported separately due to any reason, due to inefficacy and due to worsening of clinical conditions. All-cause discontinuation due to any reason combines efficacy, tolerability and other factors and can therefore be considered as a measure of 'acceptability of treatment ${ }^{16}$ or of overall 'effectiveness'.

2. Change in overall symptoms, measured by rating scales such as the Positive and Negative Syndrome Scale (PANSS) or the Brief Psychiatric Rating Scale (BPRS), or any other published scale for the assessment of overall schizophrenic symptomatology.

3. Change in positive symptoms, measured by the respective subscale of the PANSS, or the 'Scales for Assessment of Positive Symptoms' or any other published scale. 
4. Change in negative symptoms, measured by the respective subscale of the PANSS, or the 'Scales for Assessment of Negative Symptoms' or any other published scale.

5. Depressive symptoms, measured by the Calgary Depression Scale for Schizophrenia, the Hamilton Depression Rating Scale, the Montgomery Asberg Depression Scale or other published symptom scales.

6. Quality of life, measured by any published rating scale (eg, 'Heinrichs quality of life scale', 'Quality of Life Scale'.

7. Adherence, measured by any published rating scale (eg, 'Adherence Therapy Patients Satisfaction Questionnaire', 'Adherence Rating Scale').

8. Overall functioning measured by rating scales such as the Global Assessment of Functioning or the Psychosocial Performance Scale, or any other published rating scale.

9. Tolerability: adverse events. We will collect any reported adverse event that may be connected to the intervention, using a recently published classification $^{17}$ : (1) emergence of new symptoms; (2) deterioration of existing symptoms; (3) lack of improvement or deterioration of illness; (4) prolongation of treatment; (5) patient's non-compliance; (6) strains in the patient-therapist relationship; (7) very good patient-therapist relationship, therapy dependency; (8) strains or changes in family relations; (9) strains or changes in work relations; (10) any change in the life circumstances of the patient; (11) stigmatisation. Suicide attempts and any other possible adverse event related to psychosocial treatment will also be considered.

10. Mortality. We will examine this outcome in terms of (1) death for any reason, (2) death due to natural causes and (3) due to suicide.

Secondary outcomes will be measured at study endpoint. If multiple time points are given, we will take that between 26 and 52 weeks and the closest to 52 weeks.

We will give preference to the mean change from baseline to endpoint measures, and, if not available, we will take the mean values at endpoint. All continuous outcomes will be measured with rating scales that have been published in a peer-reviewed journal, because it has been shown that non-validated schizophrenia scales exaggerate differences. ${ }^{18}$ Examples of scales that we will use are the PANSS, the BPRS for overall symptoms and their respective subscales for positive and negative symptoms (see above).

\section{Search strategy}

\section{Electronic searches}

The following sources will be searched without restrictions for language or publication period: EMBASE, MEDLINE, PsycINFO, PubMed, BIOSIS and the clinical trials registers Cochrane Central Register of Controlled Trials (CENTRAL), ClinicalTrials.gov and WHO International Clinical Trials Registry Platform. A draft search strategy for PsycINFO is presented in table 1. The date of the last search update will be provided in the final publication.

\section{Reference lists and other sources}

We will also inspect previous reviews concerning psychosocial interventions for relapse prevention/maintenance treatment for schizophrenia to check if some studies meet our inclusion criteria as well. In addition, we will contact the first author of each included study published in the last 20 years for missing information about their studies.

\section{Identification and selection of studies}

Studies identified through electronic and manual searches will be listed with citation, titles and abstracts, in Citavi; duplicates will be excluded. The eligibility for inclusion process will be conducted in two separate stages: 1. Two authors will independently inspect title and abstracts identified in the literature searches, and exclude those not pertinent. Disagreement will be resolved by discussion, and where doubt still remains we will acquire the full article for further inspection and the article will proceed to the next stage.

2. Once the full articles are obtained, at least two reviewers will independently assess them for eligibility against the review criteria. If disagreement cannot be clarified by discussion, it will be resolved with a third reviewer (SL) or seeking further information from the study authors.

\section{Data extraction}

Two authors will independently extract data from all selected trials. When disagreement arises we will resolve it by discussion and, if needed, by involving a third senior author. Where this is not sufficient, we will contact the study authors.

The following data will be collected from each included study:

- Study citation, year(s) of study, registration number to trial registries, year of publication, location, setting, number of centres, sample size, diagnostic criteria, funding/sponsor.

- Methodology, including study design (type of RCT), number of arms, risk of bias (see below).

- Characteristics of study participants, including gender, age, details on diagnosis, number randomised to each arm, sociodemographic characteristics, whether psychosocial treatments naïve at baseline or with previous experience with the experimental intervention.

- Characteristics of intervention, including number and frequency of sessions, therapy setting, expertise of therapist, researcher allegiance at study arm level.

- Outcome measures, including information on whether an Intention to Treat approach has been used and how it was defined.

The two reviewers will independently input data into a Microsoft Access database specifically created for this 
Table 1 Draft search strategy for PsycINFO

\begin{tabular}{|c|c|}
\hline 2 & exp psychosis/ \\
\hline 3 & schizo\$.mp. \\
\hline 5 & $\begin{array}{l}\text { exp psychotherapy/ or exp Behavior Therapy/ or exp Cognitive Therapy/ or exp PSYCHOANALYSIS/ or exp psychotherapeutic } \\
\text { counseling/ or hypnosis/ or free association/ }\end{array}$ \\
\hline 6 & 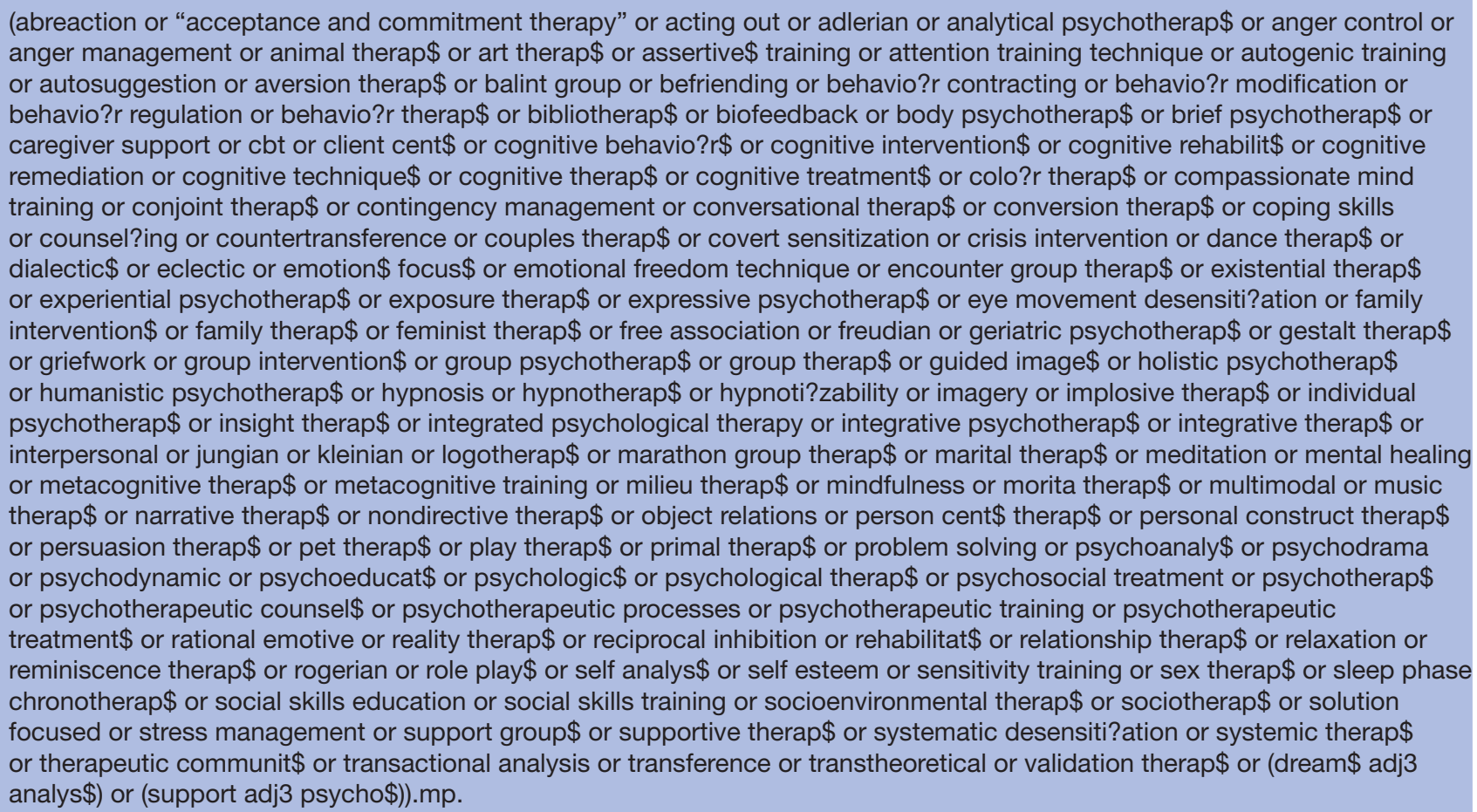 \\
\hline & or/8-11 \\
\hline 3 & 4 and 7 and 12 \\
\hline
\end{tabular}

study. The software will automatically detect any inconsistencies, and they will be resolved by discussion.

\section{Measurement of treatment effect}

\section{Relative treatment effects}

- Dichotomous outcomes: the effect size for dichotomous outcomes will be the OR and its $95 \%$ CIs.

- Continuous outcomes: for continuous outcomes we will use the standardised mean difference (SMD), because we expect that the studies can use different rating scales of schizophrenia symptomatology.

\section{Relative treatment ranking}

We will estimate the probability for each intervention to be ranked at each possible place, given the relative effect sizes as estimated in NMA. As described in Salanti et al, ${ }^{19}$ we will obtain a hierarchy of the competing interventions using the Surface Under the Cumulative Ranking curve (SUCRA) and mean ranks. SUCRA values will be expressed as percentage, showing the relative probability of an intervention to be among the best options.

\section{Dealing with missing outcome data and missing statistics}

For dichotomous outcomes, everyone allocated to the intervention will be counted whether they completed the follow-up or not. If the authors applied such a strategy, we will use their results. For our primary outcome relapse this means we will consider patients who dropped out as having relapsed in a sensitivity worst case scenario analysis, unless the authors have already included data of these patients.

For continuous outcomes we will extract data for all randomised patients if possible, and we will give preference to data based on mixed-effect models of repeated measurements of multiple imputations over lastobservation-carried-forward data. 
We will use published SDs, where available. When standard errors instead of SDs are presented, the former will be converted to SDs. ${ }^{20}$ If both are missing we will estimate SDs from $\mathrm{p}$ values or CIs, as described in Section 7.7.3 of the Cochrane Handbook for Systematic Reviews. ${ }^{21}$ If none of these options is viable, we will contact the original authors. When no information can be obtained, we will derive SDs from those of the other studies using a validated imputation technique. ${ }^{20}$

\section{Risk of bias assessment}

Risk of bias will be assessed for each included study using the revised Cochrane risk of bias tool, RoB V.2.0. ${ }^{22}$ IB and a second reviewer will independently assess the following domains:

1. Risk of bias arising from the randomisation process.

2. Risk of bias due to deviations from the intended interventions (effect of assignment to intervention).

3. Risk of bias due to missing outcome data.

4. Risk of bias in measurement of the outcome.

5. Risk of bias in selection of the reported result.

6. Overall risk of bias, as calculated by the algorithm described in Sterne et $_{\text {al. }}{ }^{22}$

Two independent review authors will assess the risk of bias in selected studies. Any disagreement will be resolved through discussion. Where necessary, the authors of the studies will be contacted for further information. Effects of studies with high risk of bias in the Overall domain will be analysed by sensitivity analyses.

\section{Data analysis}

Characteristics of the included studies

We will produce descriptive statistics and study population characteristics across all eligible trials, describing the types of comparisons and other clinical or methodological variables, such as age, duration of illness, co-medication, country, duration of study and number of sessions.

\section{Pairwise meta-analyses}

In the first step we will perform series of conventional pairwise meta-analyses by combining studies that compared the same interventions, including the comparison between active treatments and the different control arms. If very few RCTs are available or the requirements of NMA are not met, it can be that NMA will not be appropriate and, in this case, conventional pairwise meta-analysis will be applied. As heterogeneity is likely, a random-effects model will be used.

\section{Assessment of heterogeneity}

The heterogeneity (variability in relative treatment effects within the same treatment comparison) will be measured with the tau-squared (the variance of the random effects distribution). The heterogeneity variance will be assumed common across the various treatment comparisons (grouped by comparison type) and we will compare the empirical distribution with predictive distributions. ${ }^{23-25}$ Potential reasons for heterogeneity will be explored by subgroup analysis (see below).
Assessment of the transitivity assumption

Joint analysis of treatments can be misleading if the network is substantially intransitive. We assume that patients who fulfil the inclusion criteria are equally likely to be randomised to any of the interventions of interest (ie, jointly randomisable). When additional evidence of intransitivity is lacking and potential effect modifiers have similar distributions across the included studies, NMA is likely to give valid results. We will maximise the chances of transitivity in our network with regard to clinical variables by limiting our samples to participants with schizophrenia and excluding specific subgroups like acutely ill patients or patients with a comorbid disorder.

Assessment of the transitivity assumption will be done by investigating the distribution of clinical and methodological variables that can act as effect modifiers across treatment comparisons. ${ }^{26}$

These variables include administration mode and frequency of the treatment (individual/group setting, number of sessions), baseline severity and blinding (see the Investigation of heterogeneity and inconsistency section), which will also be assessed in subgroup analyses. We will investigate if these variables are similarly distributed across studies grouped by comparison.

\section{Network meta-analysis}

NMA combines direct and indirect evidence for all relative treatment effects and can therefore provide estimates with maximum power and increased precision. ${ }^{27}$ If undertaking a NMA is deemed appropriate as described above, we will conduct a random-effects NMA in a frequentist setting to synthesise all evidence for each outcome. If the estimation of the relative treatment effects is precise, then we will obtain a ranking of all treatments using the SUCRA and the mean ranks. We will assume a single heterogeneity parameter for each network. We will present the summary ORs or SMDs for all pairwise comparisons in a league table. We will also estimate the prediction intervals to assess how much the common heterogeneity affects the relative effect with respect to the extra uncertainty anticipated in a future study.

\section{Assessment of inconsistency}

The strategical and conceptual evaluation of transitivity will be supplemented with a statistical evaluation of consistency, the agreement between direct and indirect evidence.

To evaluate inconsistency, we will employ both local and global methods. ${ }^{28}$ We will employ the 'separating indirect from direct evidence approach', which separates direct evidence from indirect evidence and then evaluates their agreement to evaluate local consistency. ${ }^{29}$ We will also evaluate consistency in the entire network with the design-by-treatment interaction test. ${ }^{30}$ Tests for inconsistency are known to have low power, and empirical evidence has suggested that $10 \%$ of evidence loops published in the medical literature are expected to be inconsistent. ${ }^{31}$ Therefore, interpretation of the 
statistical inference about inconsistency will be carried out with caution and possible sources of inconsistency will be explored even in the absence of evidence for inconsistency.

\section{Investigation of heterogeneity and inconsistency}

We expect small amounts of heterogeneity and inconsistency to be present. The following potential effect modifiers of the primary outcome will be explored by subgroup analyses:

1. Studies conducted in first episode patients.

2. Studies using different definitions of relapse (if relevant).

3. Setting: individual versus group.

4. Setting: inpatients versus outpatients (at enrolment in the study).

5. Number of sessions.

6. Baseline severity (PANSS or BPRS score at baseline).

Sensitivity analyses on the primary outcome will be performed as follows:

1. Exclusion of studies in which the outcome assessor was not blind (open studies).

2. Exclusion of studies that presented only completer analyses.

3. Exclusion of studies with high risk of bias in the overall domain.

4. Exclusion of studies with researchers' allegiance. ${ }^{32-34}$

5. Patients who dropped out from the study considered as having relapsed (unless data about these patients were already considered by study authors in the outcome provided in the study).

6 . Hospitalisations and relapse defined with other criteria analysed separately.

7. Exclusion of studies where relapse or hospitalisation was not defined explicitly as primary outcome but based on our judgement.

\section{Publication bias}

To assess small study effects and publication bias, we will use funnel plots of pairwise meta-analyses if 10 or more studies are included. We will also use a comparisonadjusted funnel plot for relative treatment effects between all active and control interventions. ${ }^{35}$

\section{Statistical software}

The analysis and presentation of results will be performed using R (meta and netmeta packages).

\section{Assessment of the confidence in the evidence from NMA}

The confidence in the relative treatment effect estimated in NMA for the primary outcome will be evaluated using the Confidence in Network Meta-Analysis framework, ${ }^{28}{ }^{36}$ implemented in the web application (http:// cinema.ispm.ch/model/CINeMA_paper.pdf). This tool evaluates the credibility of the findings across the domains of within-study bias, across-study bias, indirectness, imprecision, heterogeneity and incoherence.

\section{PATIENT AND PUBLIC INVOLVEMENT}

Representatives from the Organisation 'BASTA-Bündnis für psychisch erkrankte Menschen' were involved since the stage of grant application with regular meetings. We explained them the basis of systematic reviews methodology, so that they can understand the process and provide suggestions. We asked them to provide the patients' perspective about the relevance of the topic, the choice and the importance of the appropriate outcomes. They were involved again for the preparation of this protocol, and one of them (WPH) is a co-author. We will collaborate at the preparation of a lay version of the results that will be disseminated also through the newsblog of BASTA (http://www.bastagegenstigma.de/).

\section{ETHICS AND DISSEMINATION}

Findings will be published in peer-reviewed scientific journals, granting open access and the data set will be made publicly available.

\section{Author affiliations}

${ }^{1}$ Department of Psychiatry and Psychotherapy, School of Medicine, Klinikum rechts der Isar der Technischen Universitat Munchen, Munchen, Germany

${ }^{2}$ Department of Clinical and Experimental Medicine, Institute of Psychiatry, University of Catania, Catania, Sicilia, Italy

${ }^{3}$ BASTA—Bündnis für psychisch erkrankte Menschen, Munich, Germany ${ }^{4}$ WHO Collaborating Centre for Research and Training in Mental Health and Service Evaluation, Department of Neuroscience, Biomedicine and Movement Sciences,

Section of Psychiatry, University of Verona, Verona, Italy

${ }^{5}$ Department of Health Promotion and Human Behavior, Kyoto University Graduate School of Medicine / School of Public Health, Japan, Kyoto, Japan

${ }^{6}$ Institute of Social and Preventive Medicine (ISPM), University of Bern, Bern, Switzerland

\section{Twitter Toshi A Furukawa @Toshi_FRKW}

Collaborators Samantha Roberts helped us to conduct the literature searches. Johannes Schneider-Thoma, Maximilian Huhn, Spyridon Siafis and Helena GarcíaMieres provided help and suggestions. Members of the patient organisation BASTA contributed providing the patients' perspective.

Contributors IB and SL designed this study, drafted and critically revised the protocol. GS provided substantial methodological and statistical advice. AR, GP-W, $\mathrm{CB}$ and TAF contributed with clinical and methodological input in planning the study. W-PH provided input from patients' point of view. All authors contributed to and have approved the final manuscript.

Funding This work was supported by the German Ministry for Education and Research (Bundesministerium für Bildung und Forschung, BMBF), grant number FKZ 01KG1803.

Competing interests SL has received honoraria as a consultant/advisor and/or for lectures from LB Pharma, Otsuka, Lundbeck, Boehringer Ingelheim, LTS Lohmann, Janssen, Johnson and Johnson, TEVA, MSD, Sandoz, SanofiAventis, Angelini, Sunovion, Recordati and Gedeon Richter. TAF reports personal fees from Meiji, Mitsubishi-Tanabe, MSD and Pfizer and a grant from Mitsubishi-Tanabe, outside the submitted work, and has a patent 2018-1 77688 pending.

Patient consent for publication Not required.

Ethics approval This review does not require ethical approval.

Provenance and peer review Not commissioned; externally peer reviewed.

Open access This is an open access article distributed in accordance with the Creative Commons Attribution Non Commercial (CC BY-NC 4.0) license, which permits others to distribute, remix, adapt, build upon this work non-commercially, and license their derivative works on different terms, provided the original work is properly cited, appropriate credit is given, any changes made indicated, and the use is non-commercial. See: http://creativecommons.org/licenses/by-nc/4.0/. 
ORCID iD

Irene Bighelli http://orcid.org/0000-0002-5661-5149

\section{REFERENCES}

1 Kahn RS, Sommer IE, Murray RM, et al. Schizophrenia. Nat Rev Dis Primers 2015;1:15067.

2 Hudson CG. Five-year rehospitalization experience of a state-wide cohort of persons with schizophrenia. Soc Psychiatry Psychiatr Epidemiol 2019;54:861-70.

3 Leucht S, Tardy M, Komossa K, et al. Antipsychotic drugs versus placebo for relapse prevention in schizophrenia: a systematic review and meta-analysis. The Lancet 2012;379:2063-71.

4 Bouwmans C, de SC, Mulder CL, et al. Employment and the associated impact on quality of life in people diagnosed with schizophrenia. Neuropsychiatr Dis Treat 2015;11:2125-42.

5 Chong HY, Teoh SL, DB-C W, et al. Global economic burden of schizophrenia: a systematic review. Neuropsychiatr Dis Treat 2016;12:357-73.

6 Pitschel-Walz G, Leucht S, Bauml J, et al. The Effect of family interventions on relapse and rehospitalization in schizophrenia-a meta-analysis. Schizophr Bull 2001;27:73-92.

7 Xia J, Merinder LB, Belgamwar MR. Psychoeducation for schizophrenia. Cochrane Database Syst Rev 2011;6:CD002831.

8 Zhao S, Sampson S, Xia J, et al. Psychoeducation (brief) for people with serious mental illness. Cochrane Database Syst Rev 2015;4:CD010823.

9 Jones C, Hacker D, Xia J, et al. Cognitive behavioural therapy plus standard care versus standard care for people with schizophrenia. Cochrane Database Syst Rev 2018;12:CD007964.

10 Jones C, Hacker D, Meaden A, et al. Cognitive behavioural therapy plus standard care versus standard care plus other psychosocial treatments for people with schizophrenia. Cochrane Database Syst Rev 2018;11:CD008712.

11 Hutton B, Salanti G, Caldwell DM, et al. The PRISMA extension statement for reporting of systematic reviews incorporating network meta-analyses of health care interventions: checklist and explanations. Ann Intern Med 2015;162:777-84

12 Shamseer L, Moher D, Clarke M, et al. Preferred reporting items for systematic review and meta-analysis protocols (PRISMA-P) 2015: elaboration and explanation. BMJ 2015;349:g7647.

13 Bighelli I, Salanti G, Reitmeir C, et al. Psychological interventions for positive symptoms in schizophrenia: protocol for a network metaanalysis of randomised controlled trials. BMJ Open 2018;8:e019280.

14 Egger M, Zellweger-Zähner T, Schneider M, et al. Language bias in randomised controlled trials published in English and German. The Lancet 1997;350:326-9.

15 Carpenter WT, Buchanan RW. Schizophrenia. N Engl J Med 1994;330:681-90.

16 Adams CE, Coutinho E, Davis JM, et al. Cochrane Schizophrenia Group.. In: The Cochrane library. Chichester, UK: John Wiley \& Sons Ltd, 2011.

17 Linden M, Schermuly-Haupt M-L. Definition, assessment and rate of psychotherapy side effects. World Psychiatry 2014;13:306-9.
18 Marshall M, Lockwood A, Bradley C, et al. Unpublished rating scales: a major source of bias in randomised controlled trials of treatments for schizophrenia. Br J Psychiatry 2000;176:249-52.

19 Salanti G, Ades AE, loannidis JPA. Graphical methods and numerical summaries for presenting results from multipletreatment meta-analysis: an overview and tutorial. $J$ Clin Epidemiol 2011;64:163-71.

20 Furukawa TA, Barbui C, Cipriani A, et al. Imputing missing standard deviations in meta-analyses can provide accurate results. $J$ Clin Epidemiol 2006;59:7-10.

21 editor.; .Higgins JPT. Cochrane Handbook for Systematic Reviews of Interventions. Version 5.1.0 [updated March 2011, 2011.

22 Sterne JAC, Savović J, Page MJ, et al. RoB 2: a revised tool for assessing risk of bias in randomised trials. BMJ 2019;2:14898.

23 Turner RM, Davey J, Clarke MJ, et al. Predicting the extent of heterogeneity in meta-analysis, using empirical data from the Cochrane database of systematic reviews. Int J Epidemiol 2012;41:818-27.

24 Rhodes KM, Turner RM, Higgins JPT. Empirical evidence about inconsistency among studies in a pair-wise meta-analysis. Res. Syn. Meth. 2016;7:346-70.

25 Rhodes KM, Turner RM, White IR, et al. Implementing informative priors for heterogeneity in meta-analysis using meta-regression and pseudo data. Stat Med 2016;35:5495-511.

26 Salanti G. Indirect and mixed-treatment comparison, network, or multiple-treatments meta-analysis: many names, many benefits, many concerns for the next generation evidence synthesis tool. Res. Syn. Meth. 2012;3:80-97.

27 Salanti G, Higgins JPT, Ades AE, et al. Evaluation of networks of randomized trials. Stat Methods Med Res 2008;17:279-301.

28 Salanti G, Del Giovane C, Chaimani A, et al. Evaluating the quality of evidence from a network meta-analysis. PLoS One 2014;9:e99682.

29 Dias S, Welton NJ, Caldwell DM, et al. Checking consistency in mixed treatment comparison meta-analysis. Stat Med 2010:29:932-44.

30 Higgins JPT, Jackson D, Barrett JK, et al. Consistency and inconsistency in network meta-analysis: concepts and models for multi-arm studies. Res. Syn. Meth. 2012;3:98-110.

31 Veroniki AA, Vasiliadis HS, Higgins JP, et al. Evaluation of inconsistency in networks of interventions. Int J Epidemiol 2013;42:332-45.

32 Dragioti E, Dimoliatis I, Evangelou E. Disclosure of researcher allegiance in meta-analyses and randomised controlled trials of psychotherapy: a systematic appraisal. BMJ Open 2015;5:e007206.

33 Lieb K, Osten-Sacken Jvonder, Stoffers-Winterling J, et al. Conflicts of interest and spin in reviews of psychological therapies: a systematic review. BMJ Open 2016;6:e010606.

34 Munder T, Brütsch O, Leonhart R, et al. Researcher allegiance in psychotherapy outcome research: an overview of reviews. Clin Psychol Rev 2013;33:501-11.

35 Chaimani A, Salanti G. Using network meta-analysis to evaluate the existence of small-study effects in a network of interventions. Res. Syn. Meth. 2012;3:161-76.

36 CINeMA. Confidence in network meta-analysis, 2017. Available: cinema.ispm.unibe.ch 